\title{
Research on the double slanted cantilevers of a micro structure based on Materials Mechanics
}

\author{
Chen Peng ${ }^{1, a}$, Zou Ling ${ }^{2}$, Tao $\mathrm{Yi}^{3}$, Zhang Wu${ }^{1}$, Li Yong ${ }^{1}$, Fang Guofeng ${ }^{1}$ \\ ${ }^{1}$ State Key Laboratory of NBC Protection for Civilian, Beijing, 102205, China \\ ${ }^{2}$ China Jiu Yuan Hi-tech Equipment Corporation, Beijing, 100094, China \\ ${ }^{3}$ Beijing Special Vehicle Academy, Beijing, 100072, China \\ aemail: xjy5555@qq.com
}

Keywords: Double slanted cantilevers; Mechanical Analysis; Micro Structure

\begin{abstract}
The double slanted cantilevers are used in some micro-structure designs, which have some unique characters. In this paper, the mathematical model of the double slanted cantilevers with irregular cross-section was investigated based on the theory of material mechanics. The moment of inertia and principal axes of the cross-section was derived on some hypothesis. The displacement sensitivity was also analyzed. And FEM simulations validate the calculations.
\end{abstract}

\section{Introduction}

The cantilever structure is widely used in micro-structure sensors [1], such as the accelerometer and the gyroscope used in inertia navigation system [2], and micro probe used in biochemistry detection [3]. The mechanical character of the cantilever has a close relationship with its dimensions, as well as its moment of inertia and Young's modulus, etc.

The double slanted cantilever has a good performance in a mechanical sensor [4]. But there is no detailed mathematical analysis for the double cantilevers structure.

This paper discussed the mechanical characteristics of a kind of double slanted cantilevers based on a mathematical model. The model grounds on the theory of mechanics of materials.

For the cantilevers researched in, there is a mass block suspended on the end. And the cantilevers are slanted to the same direction with a specific angle.

\section{Modeling of the double slanted cantilevers}

The model of the double slanted cantilevers with a block is established as following.

As is shown in Figure 1 (a), the big mass block, $\mathrm{CEP}_{2} \mathrm{P}_{3} \mathrm{P}_{4} \mathrm{P}_{1} \mathrm{DF}$, which can be seen as a rigid body, is suspended on the two slanted cantilevers at point $C$ and point $D$. While the two slanted cantilevers, AC and BD, which are flexible, are fixed to a base at point $\mathrm{A}$ and point $\mathrm{B}$. The point $\mathrm{O}$ is the centroid of the mass block. And the cross-section is shown in Figure 1 (b), which is an irregular hexagon, equivalent to a parallelogram cut off two identical isosceles triangles at the sharp angles.

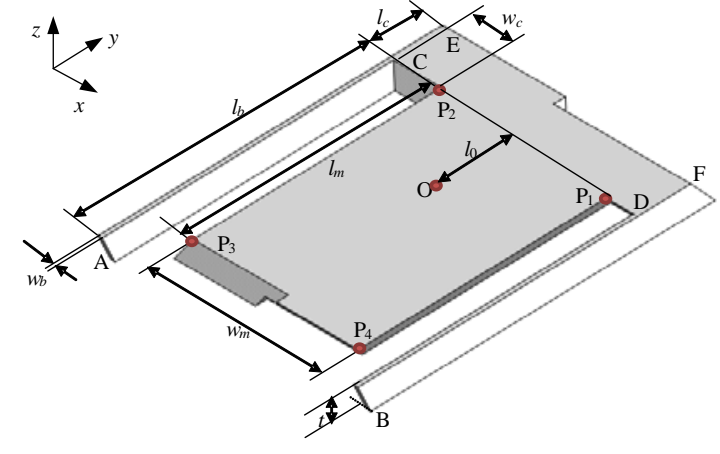

(a) Geometry of the cantilever with a block

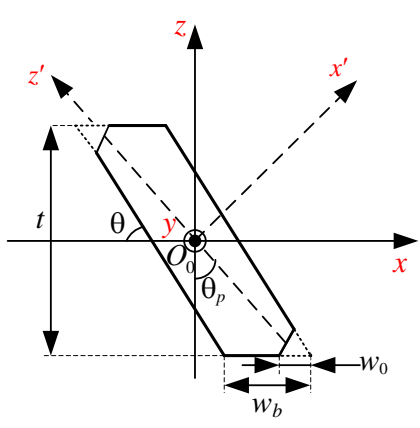

(b) the cross-section of the cantilever

Fig. 1 The draft of the slanted cantilever 
The meanings of the symbols are listed on the following table.

Table 1 the meanings of the symbols in Figure 1

$\begin{array}{clcl}w_{b} & \text { Width of one cantilever } & l_{b} & \text { Length of on cantilever } \\ w_{c} & \text { Width of the front end of the block } & l_{c} & \text { Length of the front end of the block } \\ w_{m} & \text { Width of the block } & l_{m} & \text { Length of the block } \\ l_{0} & \text { Distance from the mass center to the end of the } & t & \text { Thickness of the block } \\ & \quad \text { cantilever } & \end{array}$

As the forces on the two cantilevers are symmetrical, analysis of one cantilever is enough. As is shown in Figure 1(b), we set up two coordinate systems on the cross-section of the cantilever. The $x$-axis and $z$-axis are separately parallel to the axes in Figure 1 (a). The $z^{\prime}$-axis as well as the $x^{\prime}$-axis is the principle axis of the irregular cross-section.

According to the formula of geometry [5], we get

$$
l_{0}=\frac{l_{m} l_{m} w_{m}-l_{c}\left(w_{m}+2 w_{c}\right) l_{c}}{2\left(l_{m} w_{m}+l_{c}\left(w_{m} 2 w_{c}\right)\right)}
$$

We get the moment of inertia of the cross-section by the $\mathrm{x}$-axis by integration [5].

$$
\begin{aligned}
I_{x} & =\frac{w_{b} t^{3}}{12}-\int_{-\frac{t}{2}}^{-\frac{t}{2}+\frac{w_{0} \tan \theta}{2}} \frac{z^{2}}{\tan \theta}\left(-t+w_{0} \tan \theta-2 z\right) \mathrm{d} z-\int_{\frac{t}{2}-\frac{w_{0}}{2} \tan \theta}^{\frac{t}{2}} \frac{z^{2}}{\tan \theta}\left(-t+w_{0} \tan \theta+2 z\right) \mathrm{d} z \\
& =\frac{1}{48}\left(4 t^{3} w_{b}-6 t^{2} w_{0}{ }^{2} \tan \theta+4 t w_{0}{ }^{3} \tan ^{2} \theta-w_{0}{ }^{4} \tan ^{3} \theta\right)
\end{aligned}
$$

In the same way, we get the moment of inertia of the cross-section by the $y$-axis

$I_{z}=\frac{1}{48}\left(4 t\left(w_{b}{ }^{3}-3 w_{b} w_{0}{ }^{2}+3 w_{0}{ }^{3}\right)-6 t^{2} w_{0}{ }^{2} \cot \theta+4 t^{3} w_{b} \cot \theta^{2}-w_{0}{ }^{2}\left(6 w_{b}{ }^{2}-12 w_{b} w_{0}+7 w_{0}{ }^{2}\right) \tan \theta\right)$

And the product of inertia

$I_{x z}=\frac{1}{24}\left(t-w_{0} \tan \theta\right)\left(2 t^{2} w_{b} \cot \theta+w_{0}\left(t\left(2 w_{b}-3 w_{0}\right)-\left(w_{b}-w_{0}\right) w_{0} \tan \theta\right)\right)$

The principle axis angle $\theta_{0}$ can be obtained by solving the following equation

$$
\tan \theta_{0}=\tan \left(\frac{\pi}{2}-\theta_{p}\right)=\frac{2 I_{x z}}{I_{x}-I_{z}}
$$

The principle moment of inertia of the cross-section by the $x^{\prime}$ and $z^{\prime}$ axis can be obtained.

$$
\left\{\begin{array}{l}
I_{x^{\prime}}=\frac{I_{z}+I_{x}}{2}-\frac{\left(I_{z}-I_{x}\right) \cos 2 \theta_{p}}{2}+I_{x z} \sin 2 \theta_{p} \\
I_{z^{\prime}}=\frac{I_{z}+I_{x}}{2}+\frac{\left(I_{z}-I_{x}\right) \cos 2 \theta_{p}}{2}-I_{x z} \sin 2 \theta_{p}
\end{array}\right.
$$

When the mass block is pushed by an inertial force along the $x$-axis or $z$-axis, it will displace from its original position. The displacement of the block is determined by the movement of the point $\mathrm{C}$ and $\mathrm{D}$ on the double slanted cantilevers. Because the mass of the cantilever is very small compared to the block, the inertial force exerted on the cantilever can be ignored.

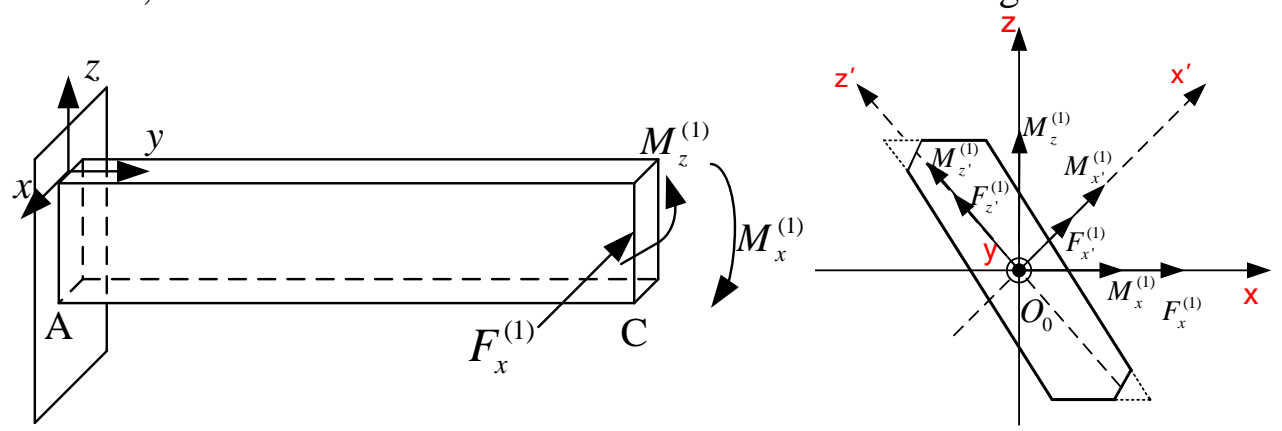

(a)The force analysis of the cantilever

(b) the force analysis of the cross-section at point $\mathrm{C}$

Fig. 2 Force analysis about an $x$-axis direction force on the cantilever 
As is shown if Figure 2, the block is suspended by two cantilevers, each cantilever endures half of the force of the block. According to Newton's second law, the force exerted on point $\mathrm{C}$ can be obtained

$$
F_{x}^{(1)}=-\frac{m_{0} a_{x}}{2}
$$

In the upper equation, $m_{0}$ is the mass of the block, and $a_{x}$ is the acceleration along the $x$-axis.

By decomposing the force and torque along the two principle axes, we get the following equations

$$
\left\{\begin{array}{c}
F_{x^{\prime}}^{(1)}=F_{x}^{(1)} \cos \theta_{p} \\
F_{z^{\prime}}^{(1)}=-F_{x}^{(1)} \sin \theta_{p}
\end{array} ;\left\{\begin{array}{c}
M_{x^{\prime}}^{(1)}=M_{x}^{(1)} \cos \theta_{p}+M_{z}^{(1)} \sin \theta_{p} \\
M_{z^{\prime}}^{(1)}=-M_{x}^{(1)} \sin \theta_{p}+M_{z}^{(1)} \cos \theta_{p}
\end{array}\right.\right.
$$

According to the laws in mechanics of materials [6], the displacement together with the torsion angle on point $\mathrm{C}$ around the principle axes is

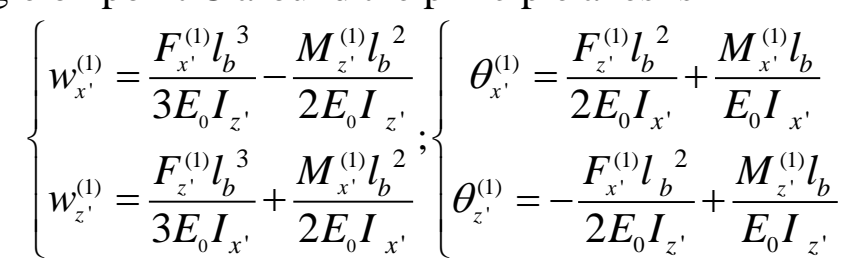

In Equation 9, the symbol $E_{0}$ represents the Young's modulus.

According the coordinate transformation rules, we can translate the displacement and the angle from the coordinate system $x^{\prime}-O_{0}-z^{\prime}$ to the coordinate system $x-O_{0}-z$ [7].

$$
\left[\begin{array}{c}
w_{x}^{(1)} \\
w_{z}^{(1)}
\end{array}\right]=\left[\begin{array}{cc}
\cos \theta_{p} & -\sin \theta_{p} \\
\sin \theta_{p} & \cos \theta_{p}
\end{array}\right]\left[\begin{array}{c}
w_{x^{\prime}}^{(1)} \\
w_{z^{\prime}}^{(1)}
\end{array}\right] ;\left[\begin{array}{c}
\theta_{x}^{(1)} \\
\theta_{z}^{(1)}
\end{array}\right]=\left[\begin{array}{cc}
\cos \theta_{p} & -\sin \theta_{p} \\
\sin \theta_{p} & \cos \theta_{p}
\end{array}\right]\left[\begin{array}{c}
\theta_{x^{\prime}}^{(1)} \\
\theta_{z^{\prime}}^{(1)}
\end{array}\right]
$$

Apply boundary conditions, $\theta_{x}^{(1)}=0, \theta_{z}^{(1)}=0$, we have the solution of the torque

$$
\left\{\begin{array}{l}
M_{x}^{(1)}=0 \\
M_{z}^{(1)}=-\frac{l_{b}}{4} m_{0} a_{x}
\end{array}\right.
$$

Take Equation 11 into Equation 9 and 10, we get the relationship of $w_{z}^{(1)}$ with the $x$-axis acceleration $a_{x}$

$$
w_{z}^{(1)}=\frac{\left(-I_{x^{\prime}}+I_{z^{\prime}}\right) l_{b}^{3} m_{0} \cos \theta_{p} \sin \theta_{p}}{24 E_{0} I_{x^{\prime}} I_{z^{\prime}}} a_{x}
$$

Analogously, when a z-axis inertial force applied on the block, we can have the force decomposition in Figure 3.
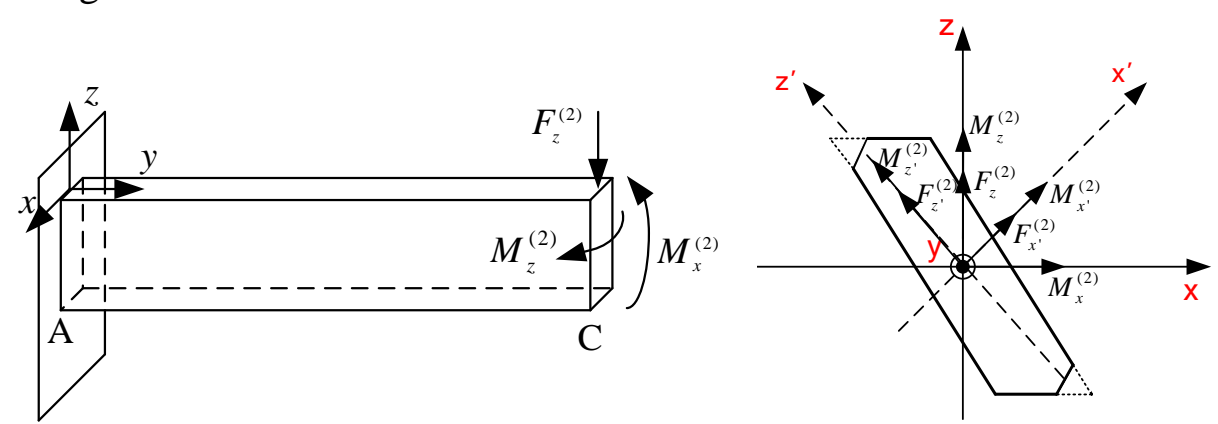

(a)The force analysis of the cantilever

(b) the force analysis of the cross-section at point $\mathrm{C}$

Fig. 3 Force analysis about a $z$-axis direction force on the cantilever

Following the same derivation methods and principles, we can get relationship of the displacement along the $z$-axis at point $\mathrm{C}$ on the slanted cantilever with the acceleration $a_{z}$, and the boundary conditions is $\theta_{z}^{(2)}=0$.

$$
w_{z}^{(2)}=\frac{m_{0} l_{b}^{2}\left(6 I_{x^{\prime}} I_{z^{\prime}}\left(8 l_{0}-5 l_{b}\right)-I_{x^{\prime}}{ }^{2} l_{b}-I_{z^{\prime}}^{2} l_{b}+\left(I_{x^{\prime}}-I_{z^{\prime}}\right)^{2} l_{b} \cos 4 \theta_{p}\right)}{192 E_{0} I_{x^{\prime}} I_{z^{\prime}}\left(I_{x^{\prime}} \cos \theta_{p}^{2}+I_{z^{\prime}} \sin \theta_{p}^{2}\right)} a_{z}
$$


At the same time, the torsion angle at point $C$ around the $x$-axis is obtained

$$
\theta_{x}^{(2)}=\frac{\left(2 l_{0}-l_{b}\right) l_{b} m_{0}}{4 E_{0}\left(I_{x^{\prime}} \cos \theta_{p}^{2}+I_{z^{\prime}} \sin \theta_{p}^{2}\right)} a_{z}
$$

In the mechanical sensor mentioned above [4], there is an electric pad faced the mass block across a thin gap. The distance from the point on the block to the electric pad is written as

$$
w_{z}(y)=d_{0}+w_{z}(0)+y \theta_{x}
$$

In Equation 15, $d_{0}$ is the original distance between the electric pad and the mass block, $y$ is the coordinate in the $y$-axis of a specific point on the block, and the origin locates where the block suspended on the cantilevers. As can be seen, $w_{z}$ is related to both translation and rotation.

The displacement sensitivity can be obtained by a differential equation

$$
S_{z}=\frac{\partial w_{z}}{\partial a}
$$

For the displacement sensitivity, it tells the relationship between the acceleration of force exerted on the block and the displacement along the $z$-axis. It's the basis of some sensor designs.

\section{FEM Simulations}

In order to validate the derivation results, FEM simulations were done using the parameters in the below table.

Table 2 the value used in the simulations and calculations

\begin{tabular}{cccccc}
\hline$w_{b}$ & $l_{b}$ & $w_{c}$ & $l_{c}$ & $w_{m}$ & $l_{m}$ \\
$0.07 \times 10^{-3} \mathrm{~m}$ & $4.15 \times 10^{-3} \mathrm{~m}$ & $0.65 \times 10^{-3} \mathrm{~m}$ & $0.77 \times 10^{-3} \mathrm{~m}$ & $2.97 \times 10^{-3} \mathrm{~m}$ & $4.37 \times 10^{-3} \mathrm{~m}$ \\
\hline$l_{0}$ & $t$ & $\theta$ & $E_{0}$ & $v$ & $\rho$ \\
$1.34 \times 10^{-3} \mathrm{~m}$ & $0.24 \times 10^{-3} \mathrm{~m}$ & $54.74^{\circ}$ & $169 \times 10^{9} \mathrm{~Pa}$ & 0.3 & $2330 \mathrm{~kg} / \mathrm{m}^{3}$ \\
\hline
\end{tabular}

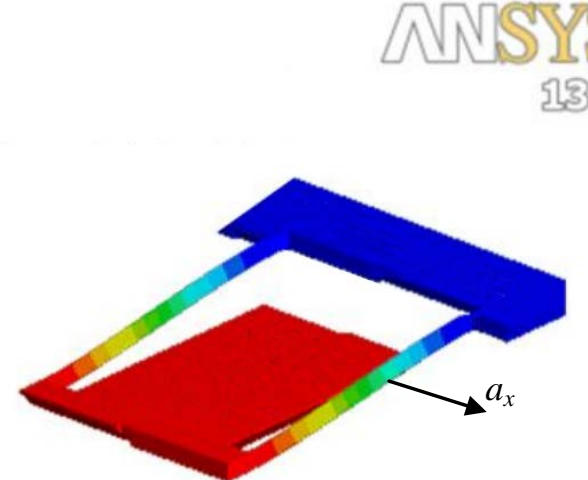

(a) The $\mathrm{x}$-axis acceleration result

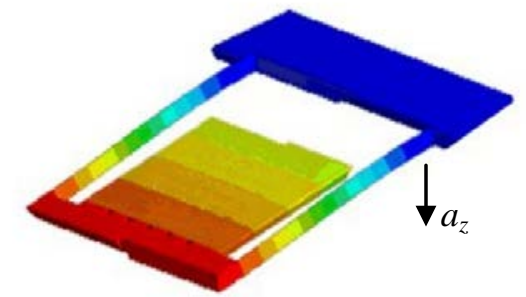

(b) the z-axis acceleration result

Fig. 4 Simulations contour plot of the structure

As is shown in Figure 4 (a), when the $x$-axis acceleration exerted on the structure, the block has a transition with the displacement in the $z$-axis in accord with Equation 12.

In Figure 4 (b), the $z$-axis acceleration will cause a transition as well as a rotation, as can be obtained in Equation 13 and 14.

Pick four points P1, P2, P3, and P4 on the block in Figure 1 to see the displacements. The contrast of the FEM simulations and the calculations based on the equations is shown in table 3 and 4.

Table 3 the $z$-axis displacement values of the points $\mathrm{P}_{1}, \mathrm{P}_{2}, \mathrm{P}_{3}$, and $\mathrm{P} 4$ by $x$-axis acceleration

\begin{tabular}{|c|c|c|}
\hline \multirow{2}{*}{ Displacement } & \multicolumn{2}{|c|}{$w_{z}^{(1)}$} \\
\cline { 2 - 3 } & Simulation value $\left(\times 10^{-8} \mathrm{~m}\right)$ & Calculation value $\left(\times 10^{-8} \mathrm{~m}\right)$ \\
\hline$d_{z}\left(\mathrm{P}_{1}\right)$ & -1.26 & -1.25725 \\
\hline$\left.d_{z} \mathrm{P}_{2}\right)$ & -1.26 & -1.25725 \\
\hline$d_{z}\left(\mathrm{P}_{3}\right)$ & -1.27 & -1.25725 \\
\hline$d_{z}\left(\mathrm{P}_{4}\right)$ & -1.27 & -1.25725 \\
\hline
\end{tabular}


Table 4 the $z$-axis displacement values of the points $\mathrm{P}_{1}, \mathrm{P}_{2}, \mathrm{P}_{3}, \mathrm{P}_{4}$ by z-axis acceleration

\begin{tabular}{|c|c|c|}
\hline \multirow{2}{*}{ Displacement } & \multicolumn{2}{|c|}{$w_{z}^{(2)}$} \\
\cline { 2 - 3 } & Simulation value $\left(\times 10^{-8} \mathrm{~m}\right)$ & Calculation value $\left(\times 10^{-8} \mathrm{~m}\right)$ \\
\hline$d_{z}\left(\mathrm{P}_{1}\right)$ & -1.24 & -1.23152 \\
\hline$d_{z}\left(\mathrm{P}_{2}\right)$ & -1.23 & -1.23152 \\
\hline$d_{z}\left(\mathrm{P}_{3}\right)$ & -0.958 & -0.89949 \\
\hline$d_{z}\left(\mathrm{P}_{4}\right)$ & -0.945 & -0.89949 \\
\hline
\end{tabular}

From the contrast, the displacement calculation results of the equations derived in the second part based on the materials mechanics were almost the same with the FEM simulations results. Therefore, the correctness of the mathematic models is validated.

\section{Conclusion}

On the basis of the theory of mechanics of materials, the mathematical model of the double slanted cantilevers was detailed investigated. The moment of inertia of the cross-section and the displacement sensitivity of the structure were derived.

The mathematic model of the double slanted structure is important for optimization design. And how each parameter of the structure influence its mechanical character is pretty distinct by analyzing the equations obtained in the paper, which laid the foundation of deeper investigations.

\section{Acknowledgement}

The work was partially supported by the Microsystems Laboratory of Science and Technology, College of Mechanical Engineering and Automation, NUDT.

The authors would like to thank Zhangqiang Hou and Dingbang Xiao for their help in research.

\section{References}

[1] A. Boisen, S. Dohn, S. S. Keller, etc. Cantilever-like micromechanical sensors [J]. Reports on Progress in Physics, 2011, 74(3), 036101.

[2] H. Rodjegård, G. I. Andersson, C. Rusu, M. Lofgren and D. Billger. Capacitive slanted-beam three-axis accelerometer: I. Modelling and design [J]. Micromech. Microeng, 2005, 15: 1989-1996.

[3] M. Nordstrom, S. Keller, M. Lillemose, etc. A. Boisen. Su-8 Cantilevers for Bio/chemical Sensing; Fabrication, Characterization and Development of Novel Read-out Methods [J]. Sensors(Basel), 2008, 8 (3): 1595-1612.

[4] Niu Zhengyi. Study on key Technologies of Monolithic Integrated Triaxial Micro-accelerometer [D]. Changsha: Graduate School of National University of Defense Technology, 2010.

[5] Shan Zhuhui. Mechanics of materials (I) [M]. Beijing: High Education Press, 2004.

[6] E. J. Mittemeijer. Fundamentals of materials science [M]. Berlin: Springer-Verlerg, 2010.

[7] G. A. Korn, T. M. Korn, Mathematical handbook for scientists and Engineers: Definitions, Theorems, and Formulas for Reference and Review [M]. Dover Publications, 2000. 